\title{
Role of tacrolimus combination therapy with mycophenolate mofetil in the prevention of organ rejection in kidney transplant patients
}

This article was published in the following Dove Press journal: International Journal of Nephrology and Renovascular Disease 2 August 2010

Number of times this article has been viewed

P Dalal'

G Shah'

D Chhabra ${ }^{2}$

Lorenzo Gallon ${ }^{2}$

'Department of Medicine, Mount Sinai Hospital, Chicago; ${ }^{2}$ Feinberg School of Medicine, Northwestern University, Chicago, Illinois, USA
Correspondence: Lorenzo Gallon Feinberg School of Medicine,

Northwestern University, 320 E Superior

Avenue, Galter 17-200

Chicago, IL 606II, USA

$\mathrm{Tel}+\mathrm{I} 3126954457$

Fax +I 3126959194

Email l-gallon@northwestern.edu
Introduction: Several new medications are now available for immunosuppression in the kidney transplant field. Tacrolimus and mycophenolate mofetil were first introduced for immunosuppression in renal transplantation in the mid 1990s. Since then, the combination of tacrolimus and mycophenolate mofetil has been evaluated in numerous clinical trials. The outcomes of these trials have varied due to differences in induction and/or maintenance therapy, drug dosing and monitoring protocols, and study design. The aim of this review is to analyze the literature critically and to provide an overview of tacrolimus and mycophenolate mofetil combination therapy in renal transplantation.

Keywords: mycophenolate mofetil, tacrolimus, kidney transplantation, immunosuppression

\section{Introduction}

With the introduction and availability of several immunosuppressive drugs with different mechanisms of action, kidney transplantation has become the treatment of choice in end-stage renal disease. Immunosuppressive drugs are given to kidney transplant recipients in two phases, ie, induction and maintenance. The induction phase usually includes short courses of various antibodies against $\mathrm{T}$ lymphocytes, along with high doses of steroids. Four classes of drugs are available for maintenance immunosuppression, ie, corticosteroids, antimetabolites (azathioprine [AZA] and mycophenolate mofetil [MMF]/mycophenolate sodium), calcineurin inhibitors (cyclosporine [CSA] and tacrolimus [TAC]), and target of rapamycin inhibitors (sirolimus [SRL] and everolimus). The aim of maintenance immunosuppression is to avoid acute rejection, interstitial fibrosis and tubular atrophy (IFTA), and to improve overall graft and patient survival. Conventional immunosuppression regimens consist of various combinations of two or three agents from different groups. These drugs act on different phases of the cell cycle to inhibit either activation or proliferation of T lymphocytes (Figure 1), which are the major mediators of acute cellular rejection. Most of the aforementioned agents have several serious side effects which are usually related to dose and duration of treatment. The rationale for combining medications from different classes is to achieve adequate immunosuppression while limiting side effects. Different transplant centers in the US use different combinations of immunosuppressive medications during the maintenance phase, and no optimal immunosuppressive strategy has been determined as yet.

In this review article, we discuss the pharmacology and pharmacokinetics of MMF and $\mathrm{TAC}$, their efficacy as maintenance immunosuppressive treatment in renal transplantation 


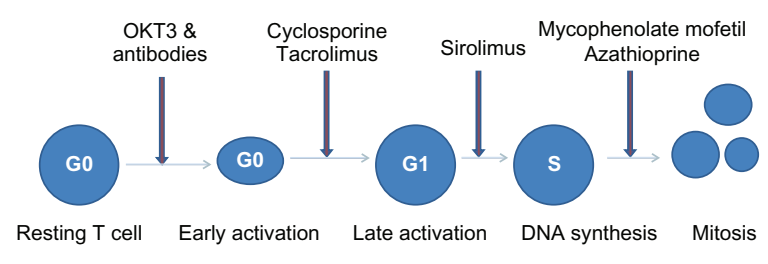

Figure I Cell cycle with site of action of immunosupreessive drugs.

compared with other commonly used immunosuppressive drug combinations, and their side effects.

\section{Pharmacology of tacrolimus}

TAC binds to an immunophilin, ie, FK binding protein. The complex of TAC-FK binding protein inhibits the phosphatase activity of calcineurin. The calcineurin enzyme is responsible for the dephosphorylation reactions required for early lymphokine gene transcription. Inhibition of calcineurin phosphatase prevents transcription activation of $\mathrm{T}$ cells, mainly via interleukin (IL)-2 and prevents the progression of T cells from the G0 to G1 phase. This results in inhibition of $\mathrm{T}$ cell proliferation in response to antigens and also generation of cytotoxic $\mathrm{T}$ cells. On a weight basis, TAC is $10-100$ times more potent then CSA in inhibiting $\mathrm{T}$ cell proliferation. ${ }^{1,2}$

\section{Pharmacokinetics of tacrolimus}

After oral administration, TAC reaches peak plasma concentration in 30 minutes to one hour. ${ }^{3}$ The bioavailability of TAC is variable, ranging from $5 \%-93 \%$, with a mean of $25 \% .{ }^{4}$ Poor water solubility of TAC and reduced gut motility in transplant recipients is responsible for the low absorption of TAC. Reduced bioavailability is reported in African-Americans, diabetics, and patients waiting for renal transplantation. ${ }^{5-12}$ Administration with food containing a moderate amount of fat can also reduce absorption and bioavailability. ${ }^{13,14}$

After absorption, TAC binds to both erythrocytes and plasma proteins. Erythrocytes bind to $75 \%-80 \%$ of the drug. Of the remaining $20 \%-25 \%$ of TAC in plasma, $99 \%$ binds to plasma proteins, mainly to albumin and alpha-1 acid glycoprotein. The distribution of TAC between plasma and erythrocytes is dependent on hematocrit, TAC concentration, and temperature. The blood concentration of TAC is about 15 times higher than the corresponding plasma concentration. Less than $0.1 \%$ of the unbound fraction of the drug is responsible for its pharmacologic activity. Measurement of blood/plasma concentration of TAC consists of both bound and unbound fractions of the drug. These are useful surrogate markers of the active drug level. ${ }^{15,16} \mathrm{TAC}$ crosses the placenta and is also excreted in breast milk. Presystemic metabolism of TAC occurs in the intestinal mucosa by cytochrome P450 (CYP) $3 \mathrm{~A} 4$ isoenzymes and P-glycoprotein. In the liver, TAC is extensively metabolized by the CYP 3A4 and CYP 3A5 isoenzymes. Polymorphisms, or genetic variations, of these isoenzymes are known to affect the dosage requirement and trough levels of TAC in stable transplant patients. ${ }^{17}$ Drugs causing either inhibition or induction of the CYP $3 \mathrm{~A}$ system may increase or decrease serum concentrations of TAC. Corticosteroids induce both CYP $3 \mathrm{~A}$ and P-glycoprotein activity and, therefore, a higher dosage of TAC is required to achieve target trough levels when used in combination with corticosteroids. ${ }^{18}$ TAC metabolites are mainly excreted via the biliary route. Less than $1 \%$ of TAC is excreted unchanged in urine and stool.

Monitoring of TAC levels is important for both safety and efficacy in transplant recipients. The lower values of the tacrolimus area under the blood concentration-time curve (AUC $<200 \mathrm{ng} / \mathrm{hr} / \mathrm{mL}$ ) on the second post-transplant day has been shown to be associated with a higher risk of acute rejection. ${ }^{16}$ A good correlation exists between the AUC and TAC concentration 12 hours postdose (trough level). ${ }^{19}$ The AUC value of $200 \mathrm{ng} / \mathrm{hr} / \mathrm{mL}$ correlates with a TAC trough level of $10 \mathrm{ng} / \mathrm{mL} .{ }^{20}$ Trough levels $>15 \mathrm{ng} / \mathrm{mL}$ have been associated with significant toxicity but without a significant reduction in acute allograft rejection. ${ }^{21}$ In the recently performed ELITE (Efficacy Limiting Toxicity Elimination) Symphony trial, a low trough level of TAC (3-7 ng/mL) was compared with low and standard doses of CSA and SRL in a quadruple regimen including daclizumab, MMF, and steroids. The low-dose TAC group had the lowest acute rejection rate with the highest glomerular filtration rate (GFR) at 12 months post-transplantation. ${ }^{22}$

\section{Pharmacology of mycophenolate mofetil}

MMF is an ester prodrug of mycophenolic acid (MPA). Two major pathways are involved in purine synthesis, ie, the de novo pathway and the salvage pathway. MPA inhibits inosine-5'-monophosphate dehydrogenase (IMPDH), the rate-limiting enzyme in the de novo pathway of purine synthesis. Therefore, it prevents the formation of guanosine monophosphate, guanine triphosphate, and deoxyguanine triphosphate, and ultimately inhibits DNA generation and cell replication. MPA is five times more potent as an inhibitor of the Type II isoform of IMPDH, which is expressed in activated $\mathrm{T}$ and $\mathrm{B}$ lymphocytes, 
than of the Type I isoform, which is expressed in most cell types. ${ }^{23}$ Due to the expression of the more susceptible form of IMPDH, MPA preferentially inhibits de novo guanosine nucleotide synthesis in lymphocytes. By preferential depletion of guanosine and deoxyguanosine nucleotides in $\mathrm{T}$ and B lymphocytes, MPA suppresses both cell-mediated immune responses and antibody formation, which are major factors in both acute and chronic allograft rejection.

In addition to inhibition of DNA synthesis in lymphocytes, depletion of guanosine nucleotides suppresses the expression of several adhesion receptors, including vascular cell adhesion molecule 1, E-selectin, and P-selectin, on vascular endothelial cells. ${ }^{24}$ This interferes with the attachment of leukocytes to endothelial cells and prevents the recruitment of lymphocytes and monocytes to sites of inflammation. ${ }^{25,26}$ Suppression of mononuclear cell recruitment is another mechanism by which MMF decreases acute and chronic graft rejection. Activation of inducible nitric oxide synthase is correlated with renal allograft rejection. Suppression of inducible nitric oxide synthase activity and nitric oxide production is presumably one of the mechanisms by which MMF prevents allograft rejection. ${ }^{27}$

\section{Pharmacokinetics of mycophenolate mofetil}

Following oral administration, MMF is absorbed rapidly and completely. It undergoes hepatic de-esterification to form MPA, an active immunosuppressant. The bioavailability of MPA from MMF is about 94\%, and reaches peak plasma concentration about two hours after oral administration. ${ }^{28}$ MPA undergoes hepatic glucuronidation to form mycophenolic acid glucuronide (MPAG), which is pharmacologically inactive. MPAG is secreted into the bile and is converted back to MPA by gut bacteria. MPA is then reabsorbed and, via hepatic recirculation, produces a second peak at 8-12 hours. ${ }^{28} \mathrm{MMF}$ is excreted in the urine as MPAG, accounting for $90 \%$ of the administered MMF dose. ${ }^{29}$ Renal failure or hemodialysis has no effect on plasma concentration of free MPA, and no dosage adjustment is required for such patients. $^{30,31}$

\section{Pharmacokinetics of mycophenolate mofetil-tacrolimus in combination}

MMF and TAC are frequently used in combination as a maintenance immunosuppressive regimen in kidney transplant recipients. The rate of absorption and systemic bioavailability of TAC remains unchanged in the presence of MMF. Therefore, no adjustment in dosing is required when TAC is coadministered with $\mathrm{MMF}^{32}$
Concomitant administration of MMF with calcineurin inhibitors affects the pharmacokinetics of MPA. CSA inhibits the enterohepatic recirculation of MPAG while TAC does not interfere with it. Therefore, the second peak in the MPA level is more pronounced in patients receiving TAC. ${ }^{33,34}$ Higher levels of MPA trough levels and AUC were observed when MMF is used in combination with TAC compared with its use in combination with CSA. ${ }^{35}$ These suggest that lower doses of MMF are required to achieve adequate immunosuppression when used with TAC, compared with CSA.

\section{Mycophenolate mofetil-tacrolimus for maintenance immunosuppression}

TAC was first discovered in 1984 as a macrolide immunosuppressant and approved by the US Food and Drug Administration in 1994 for use in organ transplantation. ${ }^{36}$ Since then, TAC has been used in different combinations in renal transplant recipients. In this review, we discuss important studies done in the field of adult kidney transplantation where the TAC-MMF combination was used as maintenance immunosuppression with or without steroids. Because clinical trials are not always designed to compare TAC-MMF with other immunosuppressive combinations, a head-to-head comparison of study outcomes is not possible.

\section{Tacrolimus-prednisone with and without mycophenolate mofetil}

Initial studies compared the efficacy of TAC-prednisone with TAC-prednisone-MMF combinations in reducing the incidence of acute rejections. More than 200 patients undergoing kidney transplantation were randomized to receive either TAC-prednisone (TAC-P, $\mathrm{n}=106$ ) or TAC-prednisone-MMF (TAC-P -MMF, $\mathrm{n}=102$ ) between September 1995 and September 1997. Mean follow-up was $15 \pm 7$ months. The rate of acute rejection was higher in the TAC-P group compared with the TAC-P-MMF group (44\% versus $27 \%, P=0.014)$. Overall patient and graft survival were similar in both groups. ${ }^{37}$ In another randomized study, the rate of acute rejection was lower at six months in the low-dose TAC-P-MMF $1 \mathrm{~g}$ or $2 \mathrm{~g}$ groups compared with a TAC-P group. Higher doses of MMF were associated with more toxicity without an improvement in efficacy. ${ }^{38}$ These studies showed that adding MMF to TAC-P reduced acute rejections and improved overall graft and patient survival.

Recently, studies have attempted to avoid or withdraw steroids early from the immunosuppressive regimen. A prospective, randomized, double-blind, placebo-controlled 
study done by Woodle et al compared the outcomes of early steroid withdrawal (day 7 post-transplant) and chronic steroid maintenance treatment using antibody induction and TAC-MMF maintenance therapy. At the end of five years, allograft survival and function were similar in both groups. Although the incidence of new-onset diabetes after transplant was similar, fewer patients in the steroid withdrawal group required insulin treatment. ${ }^{39}$ In a single-center, retrospective sequential analysis of 212 renal transplant recipients with a median follow-up of five years by Gallon et al, there was no significant difference between rate and severity of acute rejection episodes, graft survival, patient survival, and decline of renal function between the chronic steroid maintenance group $(n=96)$ and the rapid steroid elimination group $(\mathrm{n}=116)$. All patients also received induction with an IL-2 receptor antagonist and maintenance immunosuppression with TAC-MMF. Patients in the chronic steroid group had a higher incidence of hyperlipidemia and posttransplantation diabetes compared with the rapid steroid elimination group. ${ }^{40}$

\section{Tacrolimus-mycophenolate mofetil versus cyclosporine-mycophenolate mofetil}

MMF has been combined with either CSA or TAC with and without steroids for maintenance immunosuppression. Silva et al compared the safety and efficacy of a once-daily, extended-release TAC formulation with a twice-daily TAC formulation and CSA microemulsion. All patients received basiliximab induction, and concomitant MMF and steroid maintenance therapy. At one year, patient and graft survival was similar in all three groups and the TAC extended-release formulation was not inferior to twice-daily TAC dosing and CSA microemulsion in terms of biopsy-proven acute rejection, death, and graft loss. ${ }^{41}$

A retrospective analysis of the US Renal Data System containing data for 31,012 subjects was done comparing graft and recipient survival. Patients received their transplants between 01 January 1995 and 31 December 1999, with follow-up through to 31 December 2000. Three immunosuppressive regimens were analyzed, ie, prednisone-CSA-MMF (PCM, $\mathrm{n}=17,108)$, prednisone-TAC-MMF (PTM, $\mathrm{n}=7225$ ) and prednisone-CSA-AZA (PCA, $n=6679$ ). The risk of allograft failure was higher in the PTM (hazard ratio [HR] $1.09, P<0.05$ ) and PCA groups (HR 1.15, $P<0.001$ ) compared with the PCM group. Similar associations were also demonstrated in the subgroup analysis comparing transplant periods before and after 1997 in living donor transplants in adult and kidney-only recipients. ${ }^{42}$ Patient survival was similar in all three groups.

In another retrospective analysis comparing TAC $(\mathrm{n}=2393)$ and CSA microemulsion $(\mathrm{n}=4686)$ with MMF and steroids in living donor renal transplantation, the adjusted risk for graft failure at two years was significantly higher in the TAC-MMF group compared with the CSA-MMF group (HR 1.25, $P=0.013){ }^{43}$

In a prospective trial by Johnson et al, recipients of a first cadaveric renal allograft were randomly assigned to receive TAC-MMF, CSA-MMF, or TAC-AZA as immunosuppressive treatment. All patients received corticosteroids, but antibody induction was given only to the patients experiencing delayed graft function. All three groups had similar acute rejection rates and patient and graft survival at one year, but the incidence of steroid-resistant rejection requiring antithymocyte antibody was lowest in the TAC-MMF group. Posttransplant diabetes requiring insulin occurred in $14 \%$ of the TAC-AZA group, in 7\% of the CSA-MMF group, and in 7\% of the TAC-MMF group. ${ }^{44}$ At two- and three-year follow-up, all three regimens demonstrated excellent safety and efficacy. Patients treated with TAC-MMF who experienced delayed graft function had better two- and three-year allograft survival compared with patients receiving CSA-MMF (84.1\% versus $49.9 \%, P=0.02$ at three years). Patients receiving TAC with either MMF or AZA exhibited superior kidney function when compared with CSA. ${ }^{45,46}$

In the ELITE Symphony study, 1645 renal transplant recipients were randomized to receive one of four immunosuppressive regimens, ie, standard dose CSA, corticosteroids, and MMF, or daclizumab induction, MMF, and corticosteroids in combination with low-dose CSA, low-dose TAC, or low-dose SRL. The target trough levels for the low-dose CSA, low-dose TAC, and low-dose SRL groups were 50-100 ng/ $\mathrm{mL}, 3-7 \mathrm{ng} / \mathrm{mL}$, and 4-8 ng/mL, respectively. At one year, the low-dose TAC-MMF group had the highest allograft survival rates (94\% versus 89\%-93\%), highest GFR (65 mL/ min versus $57-60 \mathrm{~mL} / \mathrm{min})$, and lowest allograft rejection rates $(12 \%$ versus $24 \%-37 \%){ }^{22}$

A recent meta-analysis and metaregression compared the positive and negative effects of TAC versus CSA as primary immunosuppression in kidney transplant recipients. Data from 30 trials including 4120 patients were included in the analysis. At six months, graft loss was significantly lower in the TAC group (relative risk $0.56,95 \%$ confidence interval 0.36-0.86) and this effect persisted for up to three years. At one year, TAC-treated patients had fewer acute rejections and fewer steroid-resistant rejections, but more diabetes requiring 
insulin therapy. Rates of infections and malignancy were similar with both drugs. ${ }^{47}$

\section{Tacrolimus-mycophenolate mofetil versus sirolimus-mycophenolate mofetil}

Srinivas et al analyzed data for solitary kidney transplant recipients reported to the Scientific Registry of Renal Transplant Recipients during 2000-2005 to compare the outcomes of different immunosuppressive regimens. The combination of MMF-SRL was associated with a higher risk of acute rejection at six months post-transplantation compared with MMF-TAC $(P<0.01)$. Kaplan-Meier analysis showed inferior overall graft and patient survival with MMF-SRL compared with MMF-TAC and MMF-CSA at three years post-transplantation. ${ }^{48}$

In a randomized study comparing MMF-SRL-steroids with MMF-TAC-steroids, Larson et al did not find any difference in renal function at 12 months post-transplantation. The incidence of acute rejection was $10 \%$ in the TAC group and $13 \%$ in the SRL group $(P=0.58)$. Patient survival and graft survival were also comparable in both groups. ${ }^{49}$

\section{Tacrolimus-mycophenolate versus tacrolimus-azathioprine}

In a retrospective analysis, Schold and Kaplan compared the outcomes of TAC-MMF with TAC-AZA regimens in adult solitary kidney transplant recipients from 1998 to 2006. Overall graft loss was higher in patients treated with AZA. However, patients who received thymoglobulin induction or who were treated with the TAC-AZA combination had similar outcomes to those treated with TAC-MMF. There was no significant difference in development of malignancies, renal function, or BK virus at one year. The study was limited by its retrospective nature and the significant inequality between the number of patients in the two groups (MMF group $n=94,747$ and AZA group $n=3833) .{ }^{50}$

\section{Tacrolimus-mycophenolate mofetil versus tacrolimus-sirolimus}

Gralla and Wiseman analyzed data for 518 primary kidney transplant recipients at the University of Colorado Transplant Program during 2000-2006. Graft and patient survival, acute rejection episodes, and one-year GFR were compared between the TAC-MMF $(\mathrm{n}=211)$ and TAC-SRL $(\mathrm{n}=307)$ groups. Both groups received prednisone as part of the immunosuppressive regimen. The authors analyzed and compared outcomes for two eras of transplant, ie, 2000-2002 versus 2003-2006. One hundred and eighteen patients received TAC-SRL during 2000-2002 while 189 patients received TAC-SRL during 2003-2006. TAC-MMF was prescribed as immunosuppression to 54 and 157 patients during 2000-2002 and 2003-2006, respectively. Although induction, prednisone taper, and antimicrobial prophylaxis were similar in both eras, a higher proportion of patients in the TAC-MMF group received induction therapy. The TAC-SRL 2000-2002 group had significantly lower three-year graft and patient survival compared with the TAC-SRL 2003-2006 group and both TAC-MMF groups. A higher incidence of cardiovascular death was responsible for lower patient and graft survivals in the TAC-SRL 2000-2002 group, which improved during the 2003-2006 period, most likely as a result of aggressive pretransplant screening and treatment of asymptomatic coronary artery disease. Rates of acute rejection and BK virus nephropathy were similar, but anemia, hyperlipidemia, and new-onset diabetes were also more common at one year in the TAC-SRL group compared with the TAC-MMF group. One-year GFR was significantly lower in the TAC-SRL group compared with TAC-MMF (57.6 versus $63.1 \mathrm{~mL} / \mathrm{min}$, $P=0.008$ ) during the 2003-2006 period. The results of this study are important, but there are some limitations as well, including its retrospective nature and lack of randomization between the SRL and MMF groups, leading to a disparity in the number of patients within each group. ${ }^{51}$

In a prospective, randomized, open-label study, Gallon et al compared TAC-MMF $(n=45)$ versus TAC-SRL $(n=37)$ in a prednisone-free maintenance immunosuppression regimen. All patients received basiliximab and methylprednisone as induction therapy. At three-year follow-up, graft survival and graft function were significantly better in the TAC-MMF group compared with the TAC-SRL group. There was no difference in the rate of anemia, new-onset diabetes, and cytomegalovirus (CMV) infection between the groups. ${ }^{52}$

\section{Safety and tolerability}

The safety and tolerability of MMF was established by three pivotal studies done by the US, European, and Tricontinental renal transplant MMF study groups. MMF was generally well tolerated in most studies. While the frequency of nausea was similar, the incidences of vomiting, diarrhea, and abdominal pain were higher in patients receiving MMF compared with those receiving AZA. Most symptoms resolved with dose reduction, or interruption or withdrawal of MMF. ${ }^{53-55}$ The invasive $\mathrm{CMV}$ infection rate was also higher in patients treated with MMF compared with those treated with AZA in 
initial studies. This increased incidence was possibly related to higher endoscopic surveillance as a part of the work-up for gastrointestinal symptoms and the absence of standards for CMV prophylaxis. ${ }^{53-55}$

Although a higher dose of MMF (3 g/day) was associated with higher leukopenia at one year post-transplantation, there was no difference reported in the incidence of anemia, leukopenia, and thrombocytopenia between MMF $2 \mathrm{~g} /$ day and AZA at one year post-transplantation. The overall incidence of skin malignancy was similar for MMF $3 \mathrm{~g} /$ day and AZA groups at three years post-transplantation. There was no statistically significant difference in the incidence of skin malignancy between MMF $2 \mathrm{~g}$ /day and AZA, at one year and three years after transplantation. ${ }^{56}$

Long-term studies in renal transplant patients have suggested that CSA and TAC are equally nephrotoxic. ${ }^{57}$ The nephrotoxicity of calcineurin inhibitors is divided into acute and chronic groups. Acute oliguric tubular necrosis is a wellknown side effect of calcineurin inhibitors. This is usually caused by vasoconstriction of afferent and efferent arterioles, reduction in blood flow, and GFR. ${ }^{58}$ This acute side effect is dose-dependent, and renal function improves with cessation of therapy. ${ }^{59}$ TAC can also cause hyperkalemia, hyperuricemia, and, rarely, hemolytic uremic syndrome. ${ }^{60-63}$ Long-term use of calcineurin inhibitors is associated with IFTA. The pathologic features of IFTA include hyaline arteriolopathy, focal or striped tubulointerstitial fibrosis, and focal collapsing glomerulosclerosis. ${ }^{64}$ The chronic toxicity related to calcineurin inhibitor use is irreversible and affects long-term allograft survival. ${ }^{64}$

Some differences exist for the nonrenal side effects of CSA and TAC. Hypertension, dyslipidemia, and gingival hyperplasia are most frequently reported with CSA. The occurrence of post-transplant diabetes is higher in patients receiving TAC. Inhibition of transcription of the gene for insulin by TAC is responsible for reduced insulin secretion and occurrence of post-transplant diabetes. ${ }^{65,66}$ High TAC trough concentrations and concomitant use of steroids increase the risk of post-transplant diabetes. ${ }^{67}$ While diarrhea, vomiting, tremors, alopecia, and headache are more common with the use ofTAC, constipation and cosmetic side effects including hirsutism, are more common with CSA. ${ }^{47,68}$ Hypertrophic cardiomyopathy is also reported in pediatric transplant patients on TAC. ${ }^{69,70}$

\section{Conclusion}

Maintenance immunosuppression is necessary for all patients receiving renal transplantation, except for the rare case of identical twins. From the 1960s to the early 1980s, a combination of steroids and AZA was used to avoid allograft rejection in kidney transplant recipients. ${ }^{71}$ Introduction of CSA in the late 1980s showed better allograft survival. ${ }^{71-73}$ Subsequent addition of MMF, TAC, and SRL has allowed large numbers of combinations as maintenance immunosuppression. Combining two or three drugs from different groups is universally used to achieve adequate immunosuppression and to avoid adverse effects. In the initial studies, MMF was superior to AZA in reducing acute allograft rejection episodes at one year post-transplantation. ${ }^{53-55}$ Long-term follow-up of prospective studies and retrospective analysis of US registry data suggested reduction in late acute rejection episodes as well as reduction in IFTA independent of acute rejection episodes with MMF compared with AZA. ${ }^{74-77}$ Most of these studies were done before 2000 and used the old formulation of CSA. A recent study done by Remuzzi using the microemulsion preparation of CSA did not find any difference in the rate of acute rejection, late rejection, graft loss, and GFR between the MMF and AZA groups with or without steroids. The cost of MMF therapy was 15 times higher than that of AZA therapy. ${ }^{78,79}$ Also, a retrospective analysis by Kaplan et al suggests similar outcomes with MMF and AZA when used in combination with TAC or with appropriate induction therapy. ${ }^{50}$ The low-dose TACMMF combination had excellent overall allograft survival and better preservation of GFR, and with the lowest acute rejection rate compared with all other regimens in the ELITE Symphony trial. ${ }^{22}$ SRL in combination with a calcineurin inhibitor as a primary immunosuppressive treatment is usually associated with increased risk of graft loss and rapid decline in GFR. ${ }^{80}$ The SRL-MMF combination also has an overall higher risk of acute allograft rejection compared with TAC-MMF. ${ }^{48}$ These suggest that patients treated with SRL initially, in combination with any other medication, had an overall poor outcome. CSA and TAC are usually equally effective in combination with MMF as immunosuppressive treatment, but with differences in the side effect profile. Due to the chronic nephrotoxicity associated with calcineurin inhibitors, recent studies are attempting to replace calcineurin inhibitors with SRL several months post-transplantation. The data from the CONCEPT and Spare The Nephron studies have shown that patients switching from the MMF-calcineurin inhibitor combination to MMF-SRL post-transplantation appear to have improved renal function compared with those who continue a calcineurin inhibitor-based regimen, without an increase in acute rejection rates. ${ }^{81}$ In the unpublished data of an ongoing prospective study at Northwestern 
University, we have also found an improvement in GFR, without an increase in acute rejection rates on conversion of patients to SRL-MMF from TAC-MMF at 6-24 months post-transplantation.

In conclusion, TAC-MMF is a preferred maintenance immunosuppressive combination in adult renal transplant recipients. AZA can be used in place of MMF to reduce the cost of treatment. Switching patients from a calcineurin inhibitor to SRL several months post-transplantation appears to be an emerging option to reduce IFTA.

\section{Disclosure}

No financial support was received to carry out this research. There has been no commercial or proprietary interest in any drug, device, or equipment mentioned in the paper.

\section{References}

1. Sigal NH, Lin CS, Siekierka JJ. Inhibition of human T-cell activation by FK 506, rapamycin, and cyclosporine A. Transplant Proc. 1991;23 (2 Suppl 2):1-5.

2. Mattila PS. The actions of cyclosporin A and FK506 on T-lymphocyte activation. Biochem Soc Trans. 1996;24(1):45-49.

3. Venkataramanan R, Swaminathan A, Prasad T, et al. Clinical pharmacokinetics of tacrolimus. Clin Pharmacokinet. 1995;29(6):404-430.

4. Wallemacq PE, Furlan V, Moller A, et al. Pharmacokinetics of tacrolimus (FK506) in paediatric liver transplant recipients. Eur J Drug Metab Pharmacokinet. 1998;23(3):367-370.

5. Felipe CR, Garcia C, Moreira S, Olsen N, Silva HT, Pestana OM. Choosing the right dose of new immunossuppressive drugs for new populations: Importance of pharmacokinetic studies. Transplant Proc. 2001;33(1-2):1095-1096.

6. Fitzsimmons WE, Bekersky I, Dressler D, Raye K, Hodosh E, Mekki Q. Demographic considerations in tacrolimus pharmacokinetics. Transplant Proc. 1998;30(4):1359-1364.

7. Gruber SA, Hewitt JM, Sorenson AL, et al. Pharmacokinetics of FK506 after intravenous and oral administration in patients awaiting renal transplantation. J Clin Pharmacol. 1994;34(8):859-864.

8. Hu RH, Lee PH, Tsai MK. Clinical influencing factors for daily dose, trough level, and relative clearance of tacrolimus in renal transplant recipients. Transplant Proc. 2000;32(7):1689-1692.

9. Mancinelli LM, Frassetto L, Floren LC, et al. The pharmacokinetics and metabolic disposition of tacrolimus: A comparison across ethnic groups. Clin Pharmacol Ther. 2001;69(1):24-31.

10. Neylan JF. Racial differences in renal transplantation after immunosuppression with tacrolimus versus cyclosporine. FK506 Kidney Transplant Study Group. Transplantation. 1998;65(4):515-523.

11. Neylan JF. Effect of race and immunosuppression in renal transplantation: Three-year survival results from a US multicenter, randomized trial. FK506 Kidney Transplant Study Group. Transplant Proc. 1998;30(4):1355-1358.

12. van Duijnhoven E, Christiaans M, Schafer A, Undre N, van Hooff J. Tacrolimus dosing requirements in diabetic and nondiabetic patients calculated from pretransplantation data. Transplant Proc. 1998;30(4):1266-1267.

13. Bekersky I, Dressler D, Mekki QA. Effect of low- and high-fat meals on tacrolimus absorption following $5 \mathrm{mg}$ single oral doses to healthy human subjects. J Clin Pharmacol. 2001;41(2):176-182.

14. Jusko WJ, Piekoszewski W, Klintmalm GB, et al. Pharmacokinetics of tacrolimus in liver transplant patients. Clin Pharmacol Ther. 1995;57(3):281-290.
15. Undre NA, Stevenson P, Schafer A. Pharmacokinetics of tacrolimus: Clinically relevant aspects. Transplant Proc. 1999;31(7A):21S-24S.

16. Undre NA, van Hooff J, Christiaans M, et al. Low systemic exposure to tacrolimus correlates with acute rejection. Transplant Proc. 1999;31(1-2):296-298.

17. Haufroid V, Wallemacq P, VanKerckhove V, et al. CYP3A5 and $\mathrm{ABCB} 1$ polymorphisms and tacrolimus pharmacokinetics in renal transplant candidates: Guidelines from an experimental study. Am J Transplant. 2006;6(11):2706-2713.

18. Anglicheau D, Flamant M, Schlageter MH, et al. Pharmacokinetic interaction between corticosteroids and tacrolimus after renal transplantation. Nephrol Dial Transplant. 2003;18(11): 2409-2414.

19. BottigerY, Undre NA, Sawe J, Stevenson PJ, Ericzon BG. Effect of bile flow on the absorption of tacrolimus in liver allograft transplantation. Transplant Proc. 2002;34(5):1544-1545.

20. Mardigyan V, Giannetti N, Cecere R, Besner JG, Cantarovich M. Best single time points to predict the area-under-the-curve in long-term heart transplant patients taking mycophenolate mofetil in combination with cyclosporine or tacrolimus. J Heart Lung Transplant. 2005;24(10):1614-1618.

21. Kershner RP, Fitzsimmons WE. Relationship of FK506 whole blood concentrations and efficacy and toxicity after liver and kidney transplantation. Transplantation. 1996;62(7):920-926.

22. Ekberg H, Tedesco-Silva H, Demirbas A, et al. Reduced exposure to calcineurin inhibitors in renal transplantation. $N$ Engl J Med. 2007;357(25):2562-2575.

23. Allison AC, Eugui EM. Mycophenolate mofetil and its mechanisms of action. Immunopharmacology. 2000;47(2-3):85-118.

24. Huang HD, Liu ZH, Zhu XJ, Chen ZH, Li LS. Inhibition of mycophenolic acid on NF-kappaB activity in human endothelial cells. Acta Pharmacol Sin. 2002;23(7):649-653.

25. Raab M, Daxecker H, Karimi A, et al. In vitro effects of mycophenolic acid on the nucleotide pool and on the expression of adhesion molecules of human umbilical vein endothelial cells. Clin Chim Acta. 2001;310(1):89-98.

26. Laurent AF, Dumont S, Poindron P, Muller CD. Mycophenolic acid suppresses protein $\mathrm{N}$-linked glycosylation in human monocytes and their adhesion to endothelial cells and to some substrates. Exp Hematol. 1996;24(1):59-67.

27. Allison AC. Mechanisms of action of mycophenolate mofetil. Lupus. 2005;14 Suppl 1:S2-S8

28. Bullingham R, Monroe S, Nicholls A, Hale M. Pharmacokinetics and bioavailability of mycophenolate mofetil in healthy subjects after single-dose oral and intravenous administration. $J$ Clin Pharmacol. 1996;36(4):315-324.

29. Sweeney MJ, Hoffman DH, Esterman MA. Metabolism and biochemistry of mycophenolic acid. Cancer Res. 1972;32(9): 1803-1809.

30. Staatz CE, Tett SE. Clinical pharmacokinetics and pharmacodynamics of mycophenolate in solid organ transplant recipients. Clin Pharmacokinet. 2007;46(1):13-58.

31. de Winter BC, van Gelder T, Sombogaard F, Shaw LM, van Hest RM, Mathot RA. Pharmacokinetic role of protein binding of mycophenolic acid and its glucuronide metabolite in renal transplant recipients. J Pharmacokinet Pharmacodyn. 2009;36(6):541-564.

32. Undre NA, van Hooff J, Christiaans M, et al. Pharmacokinetics of FK 506 and mycophenolic acid after the administration of a FK 506-based regimen in combination with mycophenolate mofetil in kidney transplantation. Transplant Proc. 1998;30(4):1299-1302.

33. Shaw LM, Nawrocki A, Korecka M, Solari S, Kang J. Using established immunosuppressant therapy effectively: Lessons from the measurement of mycophenolic acid plasma concentrations. Ther Drug Monit. 2004;26(4):347-351.

34. Weber LT, Shipkova M, Lamersdorf T, et al. Pharmacokinetics of mycophenolic acid (MPA) and determinants of MPA free fraction in pediatric and adult renal transplant recipients. German Study Group on Mycophenolate Mofetil Therapy in Pediatric Renal Transplant Recipients. J Am Soc Nephrol. 1998;9(8):1511-1520. 
35. Zucker K, Rosen A, Tsaroucha A, et al. Augmentation of mycophenolate mofetil pharmacokinetics in renal transplant patients receiving Prograf and CellCept in combination therapy. Transplant Proc. 1997; 29(1-2):334-336.

36. Kino T, Hatanaka H, Hashimoto M, et al. FK-506, a novel immunosuppressant isolated from a Streptomyces. I. Fermentation, isolation, and physico-chemical and biological characteristics. J Antibiot (Tokyo). 1987;40(9):1249-1255.

37. Shapiro R, Jordan ML, Scantlebury VP, et al. A prospective, randomized trial of tacrolimus/prednisone versus tacrolimus/prednisone/ mycophenolate mofetil in renal transplant recipients. Transplantation. 1999;67(3):411-415.

38. Squifflet JP, Backman L, Claesson K, et al. Dose optimization of mycophenolate mofetil when administered with a low dose of tacrolimus in cadaveric renal transplant recipients. Transplantation. 2001;72(1):63-69.

39. Woodle ES, First MR, Pirsch J, Shihab F, Gaber AO, Van Veldhuisen P. A prospective, randomized, double-blind, placebo-controlled multicenter trial comparing early ( 7 day) corticosteroid cessation versus long-term, low-dose corticosteroid therapy. Ann Surg. 2008;248(4):564-577.

40. Gallon LG, Winoto J, Leventhal JR, Parker MA, Kaufman DB. Effect of prednisone versus no prednisone as part of maintenance immunosuppression on long-term renal transplant function. Clin J Am Soc Nephrol. 2006;1(5):1029-1038.

41. Silva HT Jr, Yang HC, Abouljoud M, et al. One-year results with extended-release tacrolimus/MMF, tacrolimus/MMF and cyclosporine/ MMF in de novo kidney transplant recipients. Am J Transplant. 2007;7(3):595-608.

42. Goldfarb-Rumyantzev AS, Smith L, Shihab FS, et al. Role of maintenance immunosuppressive regimen in kidney transplant outcome. Clin J Am Soc Nephrol. 2006;1(3):563-574.

43. Bunnapradist S, Daswani A, Takemoto SK. Graft survival following living-donor renal transplantation: A comparison of tacrolimus and cyclosporine microemulsion with mycophenolate mofetil and steroids. Transplantation. 2003;76(1):10-15.

44. Johnson C, Ahsan N, Gonwa T, et al. Randomized trial of tacrolimus (Prograf) in combination with azathioprine or mycophenolate mofetil versus cyclosporine (Neoral) with mycophenolate mofetil after cadaveric kidney transplantation. Transplantation. 2000;69(5):834-841.

45. Ahsan N, Johnson C, Gonwa T, et al. Randomized trial of tacrolimus plus mycophenolate mofetil or azathioprine versus cyclosporine oral solution (modified) plus mycophenolate mofetil after cadaveric kidney transplantation: Results at 2 years. Transplantation. 2001;72(2):245-250.

46. Gonwa T, Johnson C, Ahsan N, et al. Randomized trial of tacrolimus + mycophenolate mofetil or azathioprine versus cyclosporine + mycophenolate mofetil after cadaveric kidney transplantation: Results at three years. Transplantation. 2003;75(12):2048-2053.

47. Hardinger KL, Koch MJ, Brennan DC. Current and future immunosuppressive strategies in renal transplantation. Pharmacotherapy. 2004;24(9):1159-1176.

48. Srinivas TR, Schold JD, Guerra G, EaganA, BucciCM, Meier-KriescheHU. Mycophenolate mofetil/sirolimus compared to other common immunosuppressive regimens in kidney transplantation. Am J Transplant. 2007;7(3):586-594.

49. Flechner SM, Kurian SM, Solez K, et al. De novo kidney transplantation without use of calcineurin inhibitors preserves renal structure and function at two years. Am J Transplant. 2004;4(11):1776-1785.

50. Schold JD, Kaplan B. AZA/tacrolimus is associated with similar outcomes as MMF/tacrolimus among renal transplant recipients. Am J Transplant. 2009;9(9):2067-2074.

51. Gralla J, Wiseman AC. Tacrolimus/sirolimus versus tacrolimus/mycophenolate in kidney transplantation: Improved 3-year graft and patient survival in recent era. Transplantation. 2009;87(11):1712-1719.

52. Gallon L, Perico N, Dimitrov BD, et al. Long-term renal allograft function on a tacrolimus-based, pred-free maintenance immunosuppression comparing sirolimus vs MMF. Am J Transplant. 2006;6(7):1617-1623
53. European Mycophenolate Mofetil Cooperative Study Group. Placebo-controlled study of mycophenolate mofetil combined with cyclosporin and corticosteroids for prevention of acute rejection. Lancet. 1995;345(8961):1321-1325.

54. The Tricontinental Mycophenolate Mofetil Renal Transplantation Study Group. A blinded, randomized clinical trial of mycophenolate mofetil for the prevention of acute rejection in cadaveric renal transplantation. Transplantation. 1996;61(7):1029-1037.

55. Sollinger HW. Mycophenolate mofetil for the prevention of acute rejection in primary cadaveric renal allograft recipients. U.S. Renal Transplant Mycophenolate Mofetil Study Group. Transplantation. 1995;60(3):225-232.

56. Wang K, Zhang H, Li Y, et al. Safety of mycophenolate mofetil versus azathioprine in renal transplantation: A systematic review. Transplant Proc. 2004;36(7):2068-2070.

57. Williams D, Haragsim L. Calcineurin nephrotoxicity. Adv Chronic Kidney Dis. 2006;13(1):47-55.

58. Lanese DM, Conger JD. Effects of endothelin receptor antagonist on cyclosporine-induced vasoconstriction in isolated rat renal arterioles. J Clin Invest. 1993;91(5):2144-2149.

59. Lamas S. Cellular mechanisms of vascular injury mediated by calcineurin inhibitors. Kidney Int. 2005;68(2):898-907.

60. European FK506 Multicentre Liver Study Group. Randomised trial comparing tacrolimus (FK506) and cyclosporin in prevention of liver allograft rejection. Lancet. 1994;344(8920):423-428.

61. Gerster JC, Dudler M, Halkic N, Gillet M. Gout in liver transplant patients receiving tacrolimus. Ann Rheum Dis. 2004;63(7):894-895.

62. Holman MJ, Gonwa TA, Cooper B, et al. FK506-associated thrombotic thrombocytopenic purpura. Transplantation. 1993;55(1):205-206.

63. Boctor FN. Tacrolimus (FK506) associated thrombotic thrombocytopenic purpura/hemolytic uremic syndrome in lung transplant salvage with a plasmapheresis and cyclosporin. Egypt J Immunol. 2006;13(2):95-99.

64. Nankivell BJ, Borrows RJ, Fung CL, O’Connell PJ, Chapman JR, Allen RD. Calcineurin inhibitor nephrotoxicity: Longitudinal assessment by protocol histology. Transplantation. 2004;78(4):557-565.

65. Tamura K, Fujimura T, Tsutsumi T, et al. Transcriptional inhibition of insulin by FK 506 and possible involvement of FK 506 binding protein-12 in pancreatic beta-cell. Transplantation. 1995;59(11):1606-1613.

66. Redmon JB, Olson LK, Armstrong MB, Greene MJ, Robertson RP. Effects of tacrolimus (FK506) on human insulin gene expression, insulin mRNA levels, and insulin secretion in HIT-T15 cells. J Clin Invest. 1996;98(12):2786-2793

67. Pirsch JD, Miller J, Deierhoi MH, Vincenti F, Filo RS. A comparison of tacrolimus (FK506) and cyclosporine for immunosuppression after cadaveric renal transplantation. FK506 Kidney Transplant Study Group. Transplantation. 1997;63(7):977-983.

68. Webster AC, Woodroffe RC, Taylor RS, Chapman JR, Craig JC. Tacrolimus versus ciclosporin as primary immunosuppression for kidney transplant recipients: Meta-analysis and meta-regression of randomised trial data. BMJ. 2005;331(7520):810.

69. Jarzembowski TM, John E, Panaro F, et al. Reversal of tacrolimus-related hypertrophic obstructive cardiomyopathy 5 years after kidney transplant in a 6-year-old recipient. Pediatr Transplant. 2005;9(1):117-121.

70. Atkison P, Joubert G, Barron A, et al. Hypertrophic cardiomyopathy associated with tacrolimus in paediatric transplant patients. Lancet. 1995;345(8954):894-896.

71. Helderman JH, Van Buren DH, Amend WJ Jr, Pirsch JD. Chronic immunosuppression of the renal transplant patient. J Am Soc Nephrol. 1994;4 Suppl 8:S2-S9.

72. The Canadian Multicentre Transplant Study Group. A randomized clinical trial of cyclosporine in cadaveric renal transplantation. Analysis at three years. N Engl J Med. 1986;314(19):1219-1225.

73. Ponticelli C, Minetti L, Di Palo FQ, et al. The Milan clinical trial with cyclosporine in cadaveric renal transplantation. A three-year follow-up. Transplantation. 1988;45(5):908-913. 
74. Mathew TH. A blinded, long-term, randomized multicenter study of mycophenolate mofetil in cadaveric renal transplantation: Results at three years. Tricontinental Mycophenolate Mofetil Renal Transplantation Study Group. Transplantation. 1998;65(11):1450-1454.

75. Meier-Kriesche HU, Steffen BJ, Hochberg AM, et al. Mycophenolate mofetil versus azathioprine therapy is associated with a significant protection against long-term renal allograft function deterioration. Transplantation. 2003;75(8):1341-1346.

76. Meier-Kriesche HU, Steffen BJ, Hochberg AM, et al. Long-term use of mycophenolate mofetil is associated with a reduction in the incidence and risk of late rejection. Am J Transplant. 2003;3(1):68-73.

77. Ojo AO, Meier-Kriesche HU, Hanson JA, et al. Mycophenolate mofetil reduces late renal allograft loss independent of acute rejection. Transplantation. 2000;69(11):2405-2409.
78. Remuzzi G, Cravedi P, Costantini M, et al. Mycophenolate mofetil versus azathioprine for prevention of chronic allograft dysfunction in renal transplantation: The MYSS follow-up randomized, controlled clinical trial. J Am Soc Nephrol. 2007;18(6):1973-1985.

79. Remuzzi G, Lesti M, Gotti E, et al. Mycophenolate mofetil versus azathioprine for prevention of acute rejection in renal transplantation (MYSS): A randomised trial. Lancet. 2004;364(9433):503-512.

80. Meier-Kriesche HU, Steffen BJ, Chu AH, et al. Sirolimus with neoral versus mycophenolate mofetil with neoral is associated with decreased renal allograft survival. Am J Transplant. 2004;4(12):2058-2066.

81. Lebranchu Y, Thierry A, Toupance O, et al. Efficacy on renal function of early conversion from cyclosporine to sirolimus 3 months after renal transplantation: Concept study. Am J Transplant. 2009; 9(5):1115-1123.

\section{Publish your work in this journal}

The International Journal of Nephrology and Renovascular Disease is an international, peer-reviewed open-access journal focusing on the pathophysiology of the kidney and vascular supply. Epidemiology, screening, diagnosis, and treatment interventions are covered as well as basic science, biochemical and immunological studies. The journal welcomes original research, clinical studies, reviews \& evaluations, expert opinion and commentary, case reports and extended reports. The manuscript management system is completely online and includes a very quick and fair peerreview system, which is all easy to use. Visit http://www.dovepress.com/ testimonials.php to read real quotes from published authors.

Submit your manuscript here: http://www.dovepress.com/international-journal-of-nephrology-and-renovascular-disease-journal 